\title{
Coupling internal atomic states in a two-component Bose-Einstein condensate via an optical lattice: Extended Mott-superfluid transitions
}

\author{
Jonas Larson* and Jani-Petri Martikainen \\ NORDITA, 10691 Stockholm, Sweden
}

(Dated: November 6, 2018)

\begin{abstract}
An ultracold gas of coupled two-component atoms in an optical field is studied. Due to the internal two-level structure of the atoms, three competing energy terms exist; atomic kinetic, atomic internal, and atom-atom interaction energies. A novel outcome of this interplay, not present in the regular Bose-Hubbard model, is that in the single band and tight binding approximations four different phases appear: Two superfluid and two Mott phases. When passing through the critical point between the two superfluid or the two Mott phases, a swapping of the internal atomic populations takes place. By means of the strong coupling expansion, we find the full phase diagram for the four different phases.
\end{abstract}

PACS numbers: 37.10.Jk,05.30.Jp,03.75.Mn,03.75.Lm

\section{INTRODUCTION}

Ultracold atoms in optical lattices provide a testbed of strongly interacting many-body systems. The advantages of these systems compared to corresponding models in condensed matter physics lie in the high controllability in terms of purity, parameters, state preparation, and state detection [1]. Since the seminal experiment by Bloch and co-workers, where the Mott-superfluid phase transition was first realized [2], numerous experimental achievements have been accomplished. For example, Anderson localization of matter waves [3], the Tonks gas characterized by strong atom-atom interaction [4], and the Mott phase of two fermionic compounds [5].

Systems composed of atoms with internal level structure, such as spinor gases, yield other interesting possibilities. It has been demonstrated that the additional internal degree of freedom gives rise to novel phases and quantum phase transitions [6]. Experiments on spinor condensates include, for example, coherent transport in optical lattices [7], spin-mixing [8], inherent spin tunneling [9], and symmetry breaking [10]. In these works, as for mixtures of atomic species in optical lattices [11], direct coupling between the internal states is not considered. Coupling between the internal atomic states may indeed render new phenomena. Krutitsky et al. studied a $\Lambda$ configuration for the atoms, coupled by two optical lattices [12, 13]. They particularly showed that the Mott-superfluid phase transition may be of first order and that ferromagnetic and antiferromagnetic types of superfluid states can exist in such coupled models. Later in Ref. 14], Garcìa-Ripoll and co-workers considered individual lattice configurations for internal (dressed) atomic states. Coupling between atoms in these two lattices was induced by atom collisions. In an earlier contribution [15], we demonstrated an inherent topological phase transition in fermionic systems originating from the interplay

*Electronic address: jolarson@kth.se between internal and external atomic degrees of freedom rather than kinetic and atom-atom interaction energies as is normally the case for cold atoms in optical lattices.

In this paper we examine a gas of ultracold interacting bosonic $\Lambda$-atoms in an one dimensional optical lattice. The two atomic transitions are driven by one laser field rendering the optical lattice and another external laser lacking any spatial dependence in its mode profile. The largely detuned excited atomic level is adiabatically eliminated resulting in an effective coupled $2 \times 2$ model. An essential point of the resulting Hamiltonian is that decoupling of the two equations into two separate Schrödinger (or Gross-Pitaevskii) equations is only possible in adiabatic or diabatic regimes. The novel physics, however, is found in the intermediate regime, which will be analyzed in this work. In the next Section we show that the spectrum of the single particle Hamiltonian possesses several interesting features, such as anomalous dispersions with multiple local minima. The many-body Hamiltonian is derived in Sec. III using an expansion of the atom field operators in the lowest band Wannier functions. The magnitudes of the Hamiltonian parameters, obtained from overlap integrals containing the numerically computed Wannier functions, allow us to collect the significant terms. In the parameter regimes studied in this contribution, we end up with a Bose-Hubbard Hamiltonian, where the boson operators create or annihilate atoms in certain superpositions of their internal states. Utilizing the strong coupling expansion, we are able to find the system phase diagram in Sec. IVA.

In contrast to the usual tight-binding Bose-Hubbard model, our system possesses four different phases: two superfluid states and two Mott states characterized by different collective atomic population inversions. Moreover, the two superfluid phases have either zero or $\pi$ phase modulation between neighboring sites, giving them a ferro- or antiferromagnetic property. It is demonstrated that time-of-flight measurements would provide the information needed to distinguish between the two superfluid states as well as between the Mott states and the superfluids, while the two Mott states can be identified via 
state dependent detection.

\section{SINGLE PARTICLE HAMILTONIAN}

In order to obtain a many-body theory, we first consider properties of the corresponding single particle Hamiltonian. This enables us to systematically derive the many-body counterpart which includes atom-atom interaction in the next Section.
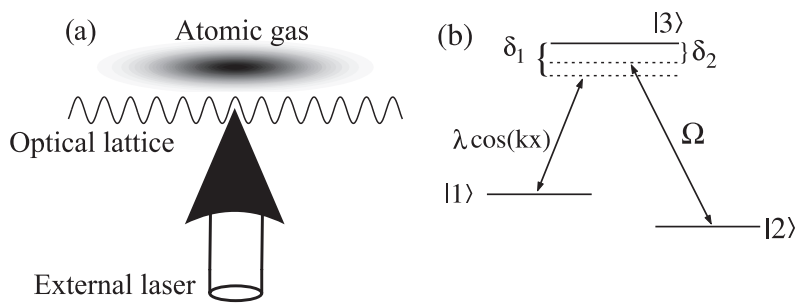

FIG. 1: Schematic configuration of system setup (a) and atom-laser configuration (b). The optical lattice drives the $|1\rangle \leftrightarrow|3\rangle$ atomic transition and the external laser the $|2\rangle \leftrightarrow$ $|3\rangle$ transition.

\section{A. The model system}

We consider an ultracold three-level $\Lambda$-atom with mass $m$ and internal levels $|i\rangle, i=1,2,3$, where $|1\rangle$ and $|2\rangle$ are the two lower metastable states and $|3\rangle$ the excited state. The atom moves in the presence of an one dimensional optical lattice which couples the two atomic states 1 and 3 with an effective coupling $\lambda$. The states 2 and 3 are coupled via an "external" field with amplitude $\Omega$, which is furthermore assumed constant over the extent of the atomic sample. Figure1 1etails the system setup and the laser-atom configuration we envision. Center-of-mass position and momentum are given by $\hat{\tilde{x}}$ and $\hat{\tilde{p}}$ respectively. Both atomic transitions are presumed highly detuned, with detunings $\delta_{1}$ and $\delta_{2}$ respectively, such that the excited state $|3\rangle$ can be adiabatically eliminated resulting in an effective two-level model of the internal states $|1\rangle$ and $|2\rangle$. Following standard procedures [16], we derive the effective Hamiltonian

$$
\hat{H}_{s p}=\frac{\hat{\tilde{p}}^{2}}{2 m}+\frac{\hbar \tilde{\Delta}}{2} \hat{\sigma}_{z}-\hbar \tilde{U}_{1} \cos (2 k \hat{x}) \hat{\sigma}_{11}+\hbar \tilde{U} \cos (k \hat{x}) \hat{\sigma}_{x},
$$

where $\tilde{\Delta}=\left|\delta_{1}-\delta_{2}\right|-\Omega^{2} / \delta_{2}-\lambda^{2} / 2 \delta_{1}$ is an effective detuning taking into account for the constant Stark shifts of states 1 and $2, \tilde{U}_{1}=\lambda^{2} / 2 \delta_{1}, \tilde{U}=\lambda \Omega\left(1 / 2 \delta_{1}+1 / 2 \delta_{2}\right)$, $k$ is the wave number of the optical lattice, and for the $\sigma$ operators we have $\hat{\sigma}_{z}=|2\rangle\langle 2|-| 1\rangle\left\langle 1\left|, \hat{\sigma}_{x}=\right| 1\right\rangle\langle 2|+| 2\rangle\langle 1|$ and $\hat{\sigma}_{22}=|2\rangle\langle 2|$. Note that the amplitudes $\tilde{\Delta}, \tilde{U}_{1}$ and $\tilde{U}$ of the last three terms of (11) can be tuned independently within the validity regime of the adiabatic elimination.
For brevity, in the following we will use dimensionless parameters. Letting $k^{-1}$ and $E_{r}=\frac{\hbar^{2} k^{2}}{2 m}$ set characteristic length and energy scales, we scale the variables as

$$
\hat{x}=k \hat{\tilde{x}}, \quad \Delta=\frac{\hbar \tilde{\Delta}}{E_{r}}, \quad U_{1}=\frac{\hbar \tilde{U}_{1}}{E_{r}}, \quad U=\frac{\hbar \tilde{U}}{E_{r}} .
$$

In terms of scaled variables and in the $|1\rangle=\left[\begin{array}{l}0 \\ 1\end{array}\right]$ and $|2\rangle=\left[\begin{array}{l}1 \\ 0\end{array}\right]$ nomenclature, Eq. (1) becomes

$$
\hat{H}_{s p}=-\frac{\partial^{2}}{\partial x^{2}}+\left[\begin{array}{cc}
\frac{\Delta}{2} & U \cos (\hat{x}) \\
U \cos (\hat{x}) & -\frac{\Delta}{2}-U_{1} \cos (2 \hat{x})
\end{array}\right] .
$$

It is worth emphasizing that with general parameters, this Hamiltonian cannot be separated into two periodic Schödinger equations. There is no $x$-independent unitary matrix that would diagonalize the $2 \times 2$ matrix of (3). Thereby, the corresponding matrix will not commute with the kinetic energy term, causing non-diagonal couplings of the transformed Hamiltonian. Indeed, this fact is a fundamental property for the results presented in this work. This non-separability is different from most earlier works on multi-component atoms in optical lattices, e.g. in Ref. [14] a rotation decouples the system and it is the atom-atom interaction that drives the coupling between two effective equations, while in Ref. [12] the atoms reside in a dark state.

The Hamiltonian (3) is periodic with period $\lambda=2 \pi$ and thus, the operator $\hat{T}=\mathrm{e}^{ \pm i \lambda \hat{p}}$ is a constant of motion. Moreover, the simultaneous inversion-displacement operator

$$
\hat{I}=\hat{\sigma}_{z} \mathrm{e}^{ \pm i \frac{\lambda}{2} \hat{p}}
$$

defines another symmetry of the Hamiltonian, where we explicitly have $\hat{I}^{2}=\hat{T}[17$. This additional invariant reveals that the spectrum is most properly described within an extended Brillouin zone with quasi momenta between -1 and 1. This property has been discussed in greater detail in Refs. [15, 17].

\section{B. Spectrum}

Labeling the momentum eigenstates by $|q\rangle(\hat{p}|q\rangle=$ $q|q\rangle$ ), it is appropriate to divide the bare basis states into two sets

$$
\begin{aligned}
& \left|\varphi_{\eta}(q)\right\rangle= \begin{cases}|q+\eta\rangle|1\rangle & \eta \text { even } \\
|q+\eta\rangle|2\rangle & \eta \text { odd }\end{cases} \\
& \left|\phi_{\eta}(q)\right\rangle= \begin{cases}|q+\eta\rangle|2\rangle & \eta \text { even } \\
|q+\eta\rangle|1\rangle & \eta \text { odd }\end{cases}
\end{aligned}
$$

where $\eta$ is any integer and $q \in(-1,1]$. Due to the symmetry defined by $\hat{I}$, the Hamiltonian (3) is on block-diagonal 
form within these states and consequently does not couple basis states of different sets; $\left\langle\varphi_{\eta^{\prime}}\left(q^{\prime}\right)|\hat{H}| \phi_{\eta}(q)\right\rangle=0$. This does not mean that the two-level structure of Eq. (3) has been decoupled, but is merely a choice of basis states (5). The analysis of Ref. [15] was carried out by taking into account for the possibility of populating both sets (5) simultaneously. Here we restrict ourself to one of the blocks of the Hamiltonian, namely discard the states $\left|\phi_{\eta}(q)\right\rangle$. Physically this implies that we assume a particular initial state of the atom. More precisely, assuming the atom to be ultracold with a momentum within the lowest Bloch band and initial internal state $|1\rangle$. Such a constrain on the initial atomic state seems reasonable within experimental feasibility. We point out though, that by limiting our investigation to a single set of (5), we do not overlook the physical phenomena of interest for this work. One important observation is that scattering between atoms may cause the two sets of basis states $\left|\varphi_{\eta}(q)\right\rangle$ and $\left|\phi_{\eta}(q)\right\rangle$ to become coupled, even if they are disconnected by the Hamiltonian. We will return to this issue in Sec. IVA.

As a periodic problem, the eigenstates of $\hat{H}$ will be of Bloch form imprinted with two quantum numbers, band index $\nu=1,2,3, \ldots$ and quasi momentum $q \in(-1,1]$,

$$
\hat{H}\left|\psi_{\nu}(q)\right\rangle=E^{\nu}(q)\left|\psi_{\nu}(q)\right\rangle,
$$

where $E^{\nu}(q)$ is the $\nu^{\prime}$ th Bloch band's dispersion curve. Due to the coupled two-level structure, the dispersions may have anomalous shapes with multiple local minima [15, 17]. This should be compared to regular energy bands, by which we mean that either $d E^{\nu}(q) / d q \geq 0$ or $d E^{\nu}(q) / d q \leq 0$ for $0 \leq q \leq 1$. Figure 2 presents several examples of the first three bands. The atypical forms of the dispersions are clearly visible. In [15] it was demonstrated how such properties of the spectrum render a topological PT for fermions and a first order PT for bosons. Whenever $U \gg U_{1}$ (plots (a)-(d)), situations similar to the ones presented in Ref. [15] are recovered. We remind that here, however, we restrict the spectrum to only one subset in Eq. (5). As was found in [15], the lowest dispersion curve can possess several local minima in this regime, which is shown in Fig. 2 (c). Note that for $\Delta \gg 0$, the regular spectrum for the lowest band is recovered, while for $\Delta \ll 0$ the spectrum is "shifted" by one unit of momentum arising from the fact that we assumed the atoms to be initially in their internal state $|1\rangle$. The reverse is obtained by considering atoms initially in $|2\rangle$ instead. Observe further that also the excited bands in the plots for large detuning possess an irregular structure.

The situation is qualitatively different if instead $\Delta, U_{1} \gg U$. Here, the internal states of the atoms are only weakly coupled and we can approximate the spectrum by consisting of free particles (atoms in internal state $|2\rangle)$ and atoms in a potential $U_{1} \cos (2 x)$ (atoms in internal state $|1\rangle)$. The corresponding two spectrums may overlap forming unusual appearances. For large $U_{1}$, the lowest dispersion curves, corresponding to $|1\rangle$-atoms, are almost flat. Thus, slight non-zero repulsive interaction between the atoms in such a band would cause an insulating state. However, the size and sign of the detuning $\Delta$ determines if the overall lowest band belongs to $|1\rangle$ or $|2\rangle$ atoms and therefore if the state is in a superfluid or a Mott state, provided repulsive atom-atom interaction. These conclusions are verified in Fig. 2 (e)-(h), where in (e) the lowest dispersion curve is approximately parabolic while in (h) it is almost flat. Similar structure of the dispersions, mixture of narrow and wide energy bands, was also encountered in honeycomb lattices [18]. We further note that anomalous lowest band dispersions can also be achieved via Bose-Hubbard models in square lattices beyond the tight binding approximation [19].

\section{THE BOSE-HUBBARD HAMILTONIAN}

One among many prototype models of many-body physics and the study of quantum PTs is the BoseHubbard model [20]. The dynamics is driven by two terms representing hopping between sites and on-site interaction between the particles. For ultracold bosonic atoms in optical lattices, the analysis is most often restricted to considering only the lowest band i.e. single band approximation and to hopping only between neighboring sites i.e. tight binding approximation. In this paper we impose these approximations, and the validity of such assumptions will be discussed in detail in Sec. IVB.

\section{A. Second quantization}

The many-body Hamiltonian is given by

$$
\begin{aligned}
\hat{H}= & \int \hat{\Psi}^{\dagger}(x) \cdot\left\{-\frac{d^{2}}{d x^{2}}+\left[\begin{array}{cc}
\frac{\Delta}{2} & U \cos (\hat{x}) \\
U \cos (\hat{x}) & -\frac{\Delta}{2}-U_{1} \cos (2 \hat{x})
\end{array}\right]\right. \\
& \left.+\frac{1}{2} \hat{\Psi}^{\dagger}(x) \cdot \mathbf{g} \cdot \hat{\Psi}(x)\right\} \cdot \hat{\Psi}(x) d x,
\end{aligned}
$$

where $\hat{\Psi}(x)$ and $\hat{\Psi}^{\dagger}(x)$ are atomic spinor annihilation and creation field operators respectively and

$$
\mathbf{g}=\left[\begin{array}{ll}
g_{11} & g_{12} \\
g_{12} & g_{22}
\end{array}\right]
$$

is the scaled onsite interaction matrix with amplitudes $g_{i j}$. We will make the approximation of assuming equal scattering amplitudes between the internal condensate states $|1\rangle$ and $|2\rangle, g_{11}=g_{22}=g$, and letting $g_{12}=0$. The atomic field operator is conveniently expressed in terms of Wannier functions as

$$
\hat{\Psi}(x)=\sum_{i} \mathrm{e}^{i \theta_{i}} \mathbf{w}_{i}(x) \hat{b}_{i}
$$



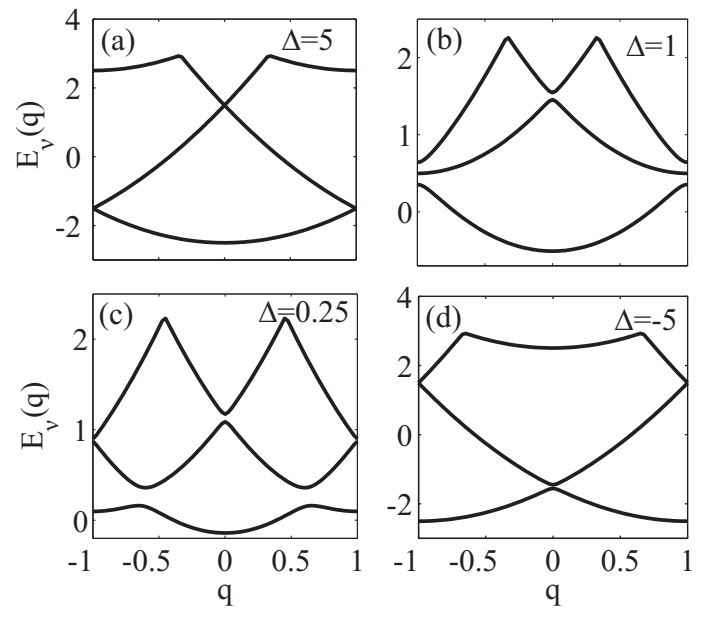
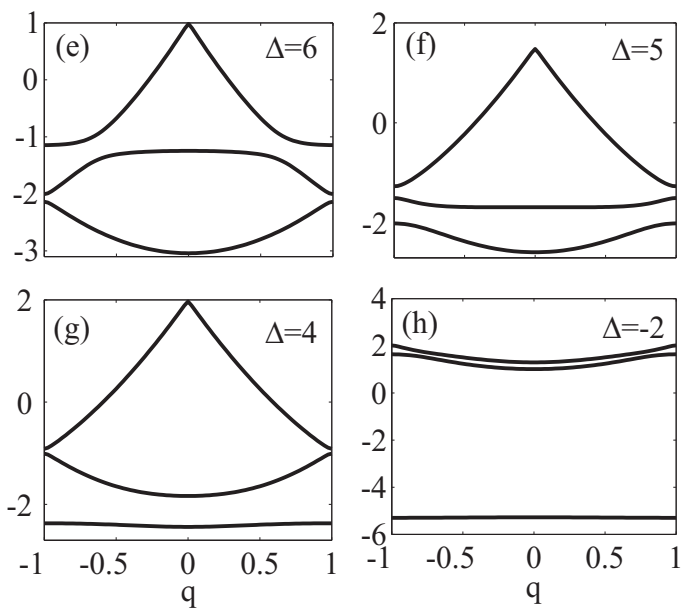

FIG. 2: Examples of the three lowest energy bands of Hamiltonian (3). In (a)-(d) we have $U>U_{1}\left(U=0.05\right.$ and $\left.U_{1}=0.025\right)$, while in (e)-(h) $U<U_{1}\left(U=0.1\right.$ and $\left.U_{1}=2\right)$. The multiple number of local minima of the lowest band is clear in (c). The large detuning case is shown in (a), in which the lowest band has the regular form. All parameters are dimensionless.

where $\mathbf{w}_{i}(x)=\left[w_{1}\left(x-x_{i}\right) w_{2}\left(x-x_{i}\right)\right]^{T}$ is the lowest band's two-component Wannier function located at $x_{i}$ and $i$ runs over all lattice sites, $\hat{b}_{i}$ is the bosonic annihilation operator for the lowest band at site $i$ and the $\theta_{i}$ 's are phases that will be determined later. We remind that we consider an one dimensional lattice so that the Wannier functions have a single spatial coordinate. From our definition of the boson operators $\hat{b}_{i}^{\dagger}$, it is clear that these have the meaning of creating an atom with wave function $\psi_{\text {atom }}\left(x-x_{i}\right)=w_{1}\left(x-x_{i}\right)|1\rangle+w_{2}\left(x-x_{i}\right)|2\rangle$. In general, both the states $|1\rangle$ and $|2\rangle$ are populated in such a state, and the populations depend highly on the system parameters. Using (9), we derive the second quantized Hamiltonian

$$
\begin{aligned}
\hat{H}_{s b}= & -\sum_{i, j} J_{i j} \hat{b}_{i}^{\dagger} \hat{b}_{j} \mathrm{e}^{i\left(\theta_{j}-\theta_{i}\right)} \\
& +\frac{1}{2} \sum_{i j k l} G_{i j k l} \hat{b}_{i}^{\dagger} \hat{b}_{j}^{\dagger} \hat{b}_{k} \hat{b}_{l}-\mu \sum_{i} \hat{n}_{i} .
\end{aligned}
$$

Here we have introduced the chemical potential $\mu$, and

$$
\begin{aligned}
& J_{i j}=-\int \mathbf{w}_{i}^{\dagger}(x) \cdot \hat{H}_{s p} \cdot \mathbf{w}_{j}(x) d x \\
& G_{i j k l}=g \int \mathbf{w}_{i}^{\dagger}(x) \cdot\left(\mathbf{w}_{j}^{\dagger}(x) \cdot \mathbf{w}_{k}(x)\right) \cdot \mathbf{w}_{l}(x) d x
\end{aligned}
$$

are the overlap integrals determining the strength of hopping and atom-atom interaction as function of the system parameters $\Delta, U_{1}$ and $U$. So far, no tight binding approximation has been applied. However, imposing the single band approximation normally motivates the use of the tight binding approximation [21], in which case one obtains a Bose-Hubbard Hamiltonian

$$
\begin{aligned}
\hat{H}_{B H}= & -J_{1} \sum_{\langle i, j\rangle} \hat{b}_{i}^{\dagger} \hat{b}_{j} \mathrm{e}^{i \theta_{j i}} \\
& +\frac{G_{0}}{2} \sum_{i} \hat{n}_{i}\left(\hat{n}_{i}-1\right)-\mu \sum_{i} \hat{n}_{i},
\end{aligned}
$$

where $\theta_{j i}=\theta_{j}-\theta_{i}$. The first sum runs over nearest neighbors, the coefficients $J_{1} \equiv J_{i i+1}$ and $G_{0} \equiv G_{i i i i}$, and $\hat{n}_{i}=\hat{b}_{i}^{\dagger} \hat{b}_{i}$. The phases $\theta_{j i}$ are chosen such that the total energy is minimized, giving

$$
\theta_{j i}= \begin{cases}0, & J_{1}>0 \\ \pi, & J_{1}<0 .\end{cases}
$$

The positions $x_{i}$ of the $i$ 'th Wannier function are not $a$ priori given in the present model. In general, the $x_{i}$ 's are taken to coincide with the minima of the effective potential. Here, however, the coupled dynamics provide a situation where well defined potentials cannot be ascribed single internal atomic states. These issues were analyzed in more detail in [15] and it was in particular found that $x_{i}=n \pi$ for any integer $n$ renders Wannier functions having familiar shapes, which for a deep lattice approximate the harmonic oscillator eigenstates. This finding may be motivated by the following argument. For positive detuning and $\Delta \gg U \gg U_{1}$, the lowest dispersion has the regular form (see Fig. 22 (a)), and since the initial atomic states are chosen to be $|1\rangle$ the effective lattice potential is in the adiabatic regime $U_{\text {eff }}(x) \propto \cos ^{2}(x)$ which possesses its minima for $x_{i}=n \pi$. Assuming a deep lattice, the Wannier functions then attain, to a good accuracy, the forms of the corresponding harmonic oscillator eigenfunctions in this limit. Note, however, that our Wannier functions are still spinors, but in this limit the $|1\rangle$ internal state is predominantly populated. We have found that 
the Wannier functions preserve their typical forms even for decreasing detunings $\Delta$ provided we pick $x_{i}=n \pi$. By this we mean that both constituent Wannier functions have an approximate Gaussian shape for relatively strong lattices. This is indeed only true for $x_{i}=n \pi$ once we have restricted the analysis to the basis set $\left|\varphi_{\eta}(q)\right\rangle$ of Eq. (5). Any other choice of $x_{i}$ results in Wannier functions with atypical shapes and being less localized. Therefore, in the following we will choose $x_{i}=n \pi$.

The anomalous form of the lowest dispersion curve implies that the nearest neighbor hopping parameter $J_{1}$ can attain both positive and negative values [12]. The coupling parameters, given by the various overlap integrals (11), are calculated using the spinor Wannier functions obtained numerically from diagonalization of the Hamiltonian (3). We display, as a function of $\Delta, J_{i}(i=1,2,3)$ in Fig. 3 (a) and (c), while (b) and (d) show $G_{0}$ and $G_{1}$, where $G_{1}=G_{i j j i}$ and $j=i \pm 1$. The coefficients $J_{2} \equiv J_{i i+2}$ and $J_{3} \equiv J_{i i+3}$ describe the next and nextnext nearest neighbor tunneling strengths. The parameters are $U=1$ and $U_{1}=0.5$ in (a) and (b), and $U=0.5$ and $U_{1}=1$ in (c) and (d). In these two examples, $\left|J_{1}\right| \gg\left|J_{i \neq 1}\right|$ outside the neighborhood where $J_{1}=0$. We note that $G_{1}$ is considerably smaller than $G_{0}$ for any $\Delta$.

Let us comment on the elaborate structure of the system. For large detunings, $\left|\delta_{1}\right| \gg \lambda$ and $|\Delta| \gg$ $\lambda, \Omega$, atoms in state $|1\rangle$ moves in an effective potential $U_{\text {eff }}(x) \propto \cos ^{2}(x)$. This is the common adiabatic dispersive situation utilized in most experiments. The effective potential in such case is obtained via adiabatic diagonalization of the Hamiltonian. It is known that such approach gives rise to non-adiabatic corrections, which can be expressed in terms of effective gauge fields 22]. However, in the dispersive regime, the non-adiabatic corrections are vanishingly small and can be neglected. On the other hand, in the intermediate regime, which we are interested in, these corrections cannot be overlooked and must be taken into account. In fact, only in the adiabatic limiting situations $|\Delta| \rightarrow \pm \infty$ can an effective potential be assigned to the internal states of the atoms [15, 23]. Only in this limit will the boson operator $\hat{b}_{i}^{\dagger}$ create an atom in a bare state $\psi_{\text {atom }}\left(x-x_{i}\right)=w_{1}\left(x-x_{i}\right)|1\rangle$ or in a bare state $\psi_{\text {atom }}\left(x-x_{i}\right)=w_{2}\left(x-x_{i}\right)|2\rangle$, otherwise both internal states are populated. Three different energy contributions drive the system, internal and kinetic atomic energies and atom-atom interaction energy. This is not evident in the second quantized formalism (12), containing only two parameters $J_{1}$ and $G_{0}$. However, the internal atomic energy is indirectly embodied in these two parameters, since the internal state greatly influences the spinor Wannier functions.

\section{MOTT-SUPERFLUID PHASES}

In the previous section we found that the hopping coefficient may change sign as $\Delta$ is varied. Thus, the ef-
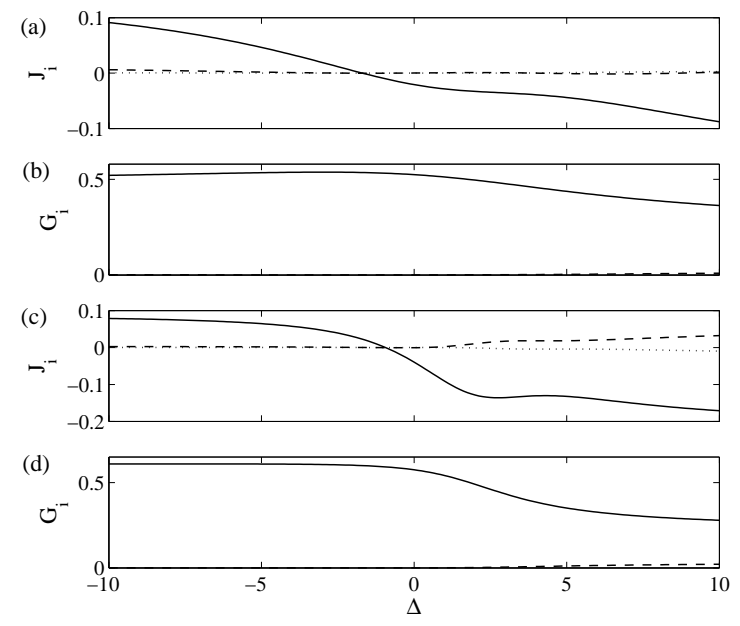

FIG. 3: Two examples of the first three hopping parameters $J_{1}$ (solid line), $J_{2}$ (dashed line) and $J_{3}$ (dotted line) as function of $\Delta$ in (a) and (c), and $G_{0}$ (solid line) and $G_{1}$ (dashed line) in (b) and (d). In both examples, $J_{1}$ changes sign. The dimensionless parameters are $g=1, U=1$, and $U_{1}=0.5$ in (a) and (b), and $g=1, U=0.5$, and $U_{1}=1$ in (c) and (d).

fective hopping, $J_{1} / G_{0}$, can be tuned from large negative to large positive values. As will be described below, the change of sign of $J_{1}$ is an outcome of a first order PT. Across this critical point, the internal atomic state changes and thus defines two different phases. In addition, in the superfluid phase, the sign of $J_{1}$ affects the character of the many-body atomic state due to the phase matching between the Wannier functions in the expansion (9).

For the phase diagrams, the sign change in $J_{1}$ implies that both positive and negative regimes for the hopping should be considered, and not restricted to only positive as in the regular Bose-Hubbard model [20]. We will present typical examples of the phase diagrams in the $\mu-\Delta$ plane rather than in the $\mu-J_{1}$ plane, since experimentally $\Delta$ is an easily controllable parameter. The phase diagrams are achieved by applying the strong coupling expansion [24], which has turned out to reproduce accurate results for the Mott boundaries of the BH model in one dimension.

\section{A. Phase diagrams}

The parameters are derived from the numerically obtained spinor Wannier functions. Moreover, $U$ and $U_{1}$ are chosen in such a way that we can safely impose the above discussed approximations (see also the following subsection).

The phase diagrams obtained for the parameters of Fig. 3 (a) and (c) are displayed in Fig. 4 (a) and (b) respectively. The plot only shows the first four Mott lobes. 

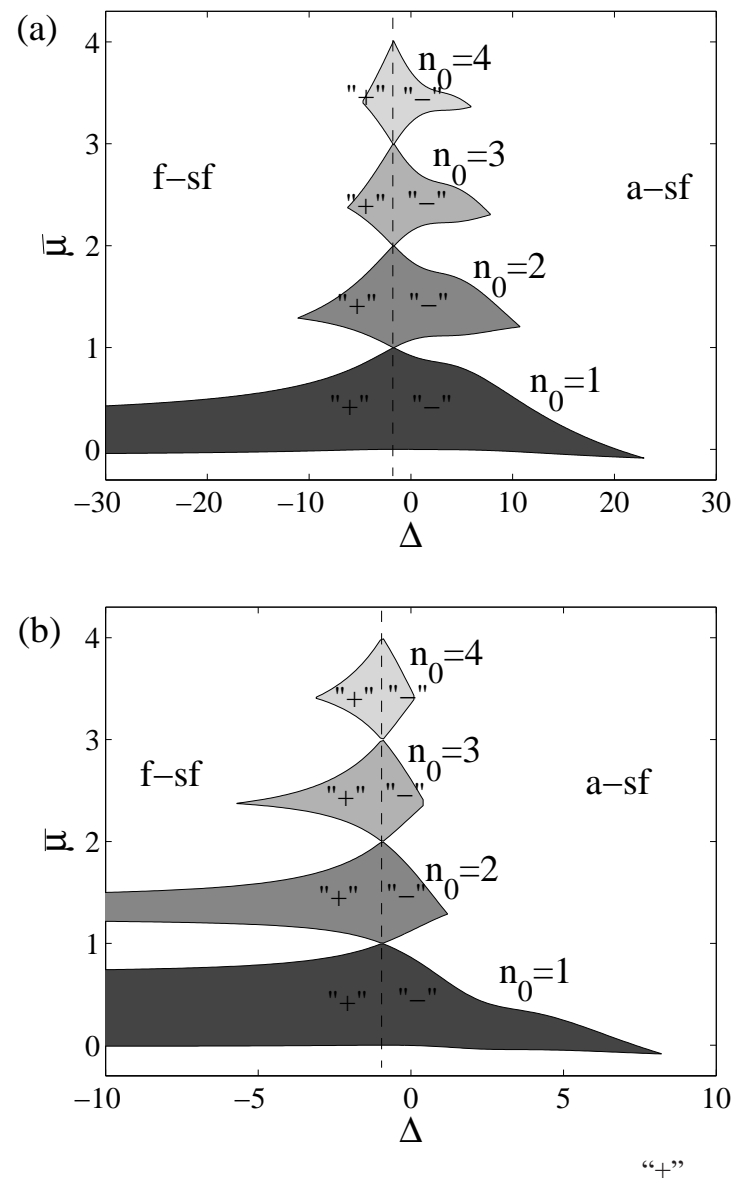

(c)
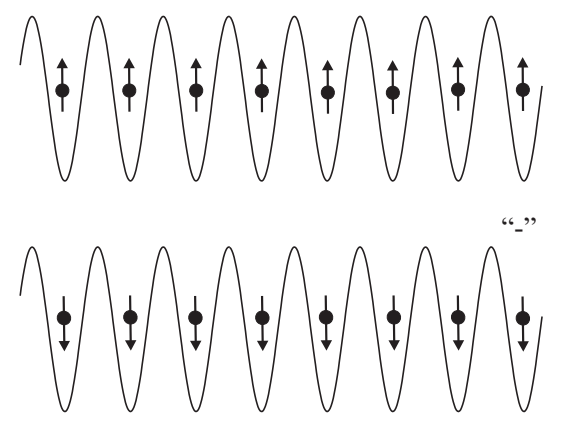

FIG. 4: Upper two plots (a) and (b) display the phase diagrams (showing the first four Mott lobes) corresponding to Fig. 3 (a) and (c). The vertical dashed lines is the phase boundary between antiferromagnetic (a-sf) or ferromagnetic superfluid states (f-sf) and between "+" and "-" Mott phases. The lower plot (c) gives a schematic picture of the "+" and "-" Mott states by describing the internal atomic states by their respective Bloch vectors.

The vertical dashed line gives the crossover between positive and negative $J_{1}$; left of the line $J_{1}>0$ and right of it $J_{1}<0$. We note the asymmetry of the Mott zones on each side of the dashed line. This irregularity originates from the Stark shift term $U_{1} \cos (2 x)$ appearing in the Hamiltonian (3), which effectively shift the resonance condition that defines the critical detuning $\Delta_{c}$. Without this term [25], the Mott lobes are symmetric with respect to the dashed line.

In order to identify the different phases of Fig. 4 (a) and (b), we first need to understand the underlying physics of the single particle Hamiltonian (3). In Ref. [15] we demonstrated that a first order $\mathrm{PT}$ is obtained in an ideal gas of coupled two-component bosonic or fermoinic atoms, when the detuning is varied across a critical value. In the case of fermions, this describes a topological PT, as the Fermi surface changes topology across the critical point; each atom changes its momenta by either \pm 1 . Changing the internal states of the atoms also shift the atomic momenta by a multiple of the unit momentum. This yields a competition between the two terms; for certain parameters it is more favorable to lower the internal atomic energies, while in other situations it is rather the atomic kinetic energy that should be minimized. When crossing the critical point for this $\mathrm{PT}$, which occurs exactly when the hopping changes sign, each atom shifts their momenta by \pm 1 and their internal state populations are swapped. Thus, the collective atomic inversion, which gives the population imbalance between the internal atomic states, works as an order parameter [15].

The atomic structure of the different phases become more clear by thinking of the two-level atoms as spin $1 / 2$ particles. The single particle wave function is written as

$$
\Phi(x)=\psi_{1}(x)|1\rangle+\psi_{2}(x)|2\rangle
$$

giving the Bloch vector components

$$
\begin{aligned}
& u \equiv\left\langle\hat{\sigma}_{x}\right\rangle=2 \operatorname{Re}\left[P_{12}\right], \\
& v \equiv\left\langle\hat{\sigma}_{y}\right\rangle=-2 \operatorname{Im}\left[P_{12}\right], \\
& w \equiv\left\langle\hat{\sigma}_{x}\right\rangle=P_{11}-P_{22},
\end{aligned}
$$

where $P_{i j}=\int \psi_{i}^{*}(x) \psi_{j}(x) d x$. The last component of the Bloch vector, $w$, is simply the atomic inversion. From the decoupling of the two basis sets (5), it follows that whenever only one bases set is populated $P_{i j}=0 \mathrm{un}$ less $i=j$. Thereby, $u=v=0$ in the "+" Mott and the "-" Mott states, and the corresponding atomic Bloch vector points either towards the north or the south pole of the Bloch sphere respectively, as schematically shown

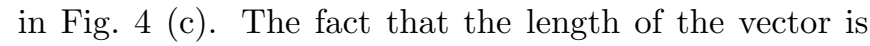
in general smaller than unity reflects the entanglement shared between internal and motional atomic degrees of freedom.

The same type of PT exists also for incommensurate fillings (superfluid states). However, contrary to atoms in the Mott state, the superfluid states possess as well an inherent coherence within the atoms and one finds that the PT manifests itself in this coherence. In particular, such transition corresponds to going from an antiferromagnetic (a-sf) to a ferromagnetic superfluid state (f-sf), or vice versa. The terms antiferro- and ferromagnetic come from phase matching between site wave functions 
in the expansion (9). For the antiferromagnetic state, there is a $\pi$-phase sign-flip between neighboring Wannier functions. We term these superfluid states a-sf or f-sf in Fig. 4. Note that the terminology of antiferro and ferromagnetic states originates from the phase-matching between Wannier functions, and not from the spin orientation (Bloch vectors) between neighboring sites.

The analysis has been carried out by restricting the atomic states to the set $\left\{\left|\varphi_{\eta}(q)\right\rangle\right\}$ of Eq. (5). For the many-body system, one must take atom-atom scattering into account. These contributions break the symmetry defined by the operator $\hat{I}$ given in Eq. (4), and consequently cause coupling between the two sets of (5). The approach utilized in this work does not include such population transfer processes. We will now argue that these are indeed very small even at very large effective scattering amplitudes $g$ (and $g_{12}$ ). To do so we solve the corresponding Gross-Pitaevskii equation (7) obtained by replacing the atomic operators by mean-field wave functions. We start from an ansatz wave function for $\Psi(x)$ which completely resides in one of the bases sets $\left\{\left|\varphi_{\eta}(q)\right\rangle\right\}$ or $\left\{\left|\phi_{\eta}(q)\right\rangle\right\}$. Given any set of parameters $U, U_{1}, \Delta$, and $\mathbf{g}$, the ground state is obtained via imaginary time-evolution. If $\mathbf{g}=0$, no population transfer occurs between the two bases sets and the obtained ground state populates only one basis set. For the field amplitudes $U$ and $U_{1}$ of Fig. 4 and various $\Delta$, effective couplings $g=100$ and $g_{12}=0$ give a population imbalance between the bases sets for the ground state of $<5 / 1000$. That is, even for effective scattering amplitudes as large as 100 recoil energies, more than $99.5 \%$ of the ground state population resides in one of the basis sets. It turns out that the amount of coupling between the two bases sets is more sensitive to the strength of $g_{12}$ than to the one of $g$. For $g_{12}=100$, about $10 \%$ may populate the orthogonal basis set (population imbalance $1 / 9)$. On the other hand, we find that $w$ does not depend on $g_{12}$, and therefore a non-zero $g_{12}$ leaves the order parameter $w$ unchanged. We may conclude that in terms of the Bloch vector, scattering between the atoms induces only a small deviation of the vector from the $z$-axis, but the projection onto the $z$-axis is very insensitive to these scattering processes. We should point out that solving the Gross-Pitaevskii equation is only justified deep in the superfluid regime, but nonetheless, we believe that the above analysis utilizing very large scattering amplitudes demonstrates the robustness of the assumption of neglecting coupling between the two bases sets (see also [26]).

In Ref. [15] we showed that the anomalous structure of the dispersions is also encountered in a two dimensional lattice. Furthermore, in [26] we studied the dynamics of the mean-field PT deriving from the atypical dispersions in both one and two dimensions, and proved its persistence in two dimensions. Thereby, it is clear that our findings are not restricted to the special case of a one dimensional lattice, but apply also to higher dimensions. However, in the present work, studying higher dimensions would be considerably more cumbersome as the Hamiltonian cannot be written as a simple sum of lattice potentials in the different spatial directions, and consequently calculation of the corresponding Bloch and Wannier functions are not as straight forward as for a regular dispersive square or cubic lattice. Moreover, the strong coupling expansion used for determining the phase diagrams in the proceeding section only provides quantitatively accurate results in one dimension, while in two and three dimensions it reproduces only qualitative results.

\section{B. Validity of approximations}

Various approximations have been imposed in order to derive the phase diagrams; tight binding and single band approximations, and truncating the strong coupling expansion at third order. In this subsection we systematically discuss the justification of such assumptions. A rule of thumb is that these approximations are all related in the regular Bose-Hubbard model and share more or less the same validity regimes 21]. It is not deducible that this holds also for our two-component model where for instance the Wannier functions are spinors.

Already Fig. 2 justify the application of the tight binding approximation. This, of course, is due to our choice of parameters. As a second check of the tight binding approximation, we have modified the strong coupling expansion to include tunneling processes beyond nearest neighbors and recalculated the phase diagrams of Fig. 4 and found only minimal corrections. Note that when $J_{1}=0$, the tight binding approximation in general fails. In this regime, however, the hopping terms beyond nearest neighbor are, for the examples presented in this paper, very small such that the dynamics is predominantly driven by the onsite interaction and therefore the system must be in a Mott state.

The justification for the use of the single band approximation has been investigated by evaluating overlap integrals between spinor Wannier functions of the first and the second band. For Fig. 4, we found that these coupling elements are everywhere much smaller than $J_{1}$ and $G_{0}$ except for $\Delta \approx 0$ in (b). Thus, tuning $\Delta$ non-adiabatically across resonance, when $U=0.5$ and $U_{1}=1$, may cause population of excited bands.

Finally, in order to discuss on what grounds the third order strong coupling expansion may be applied, we have compared the phase diagrams of Fig. 4 with the ones obtained by utilizing second order perturbation theory instead of third order and found only slight modifications.

\section{CHARACTER OF DIFFERENT PHASES}

As pointed out in the introduction, coupled two-level atoms in optical lattices were first studied in Ref. [12], 
considering two optical lattices driving a Raman transition in $\Lambda$ atoms. By tuning the relative phase between the two lattices, it was demonstrated that the hopping coefficient in the corresponding Bose-Hubbard Hamiltonian could attain negative as well as positive values. Furthermore, due to the phase factor in the Wannier expansion (9), it follows that the coherence in a superfluid state is different depending on the sign of the hopping coefficient. In particular, if the phase difference between consecutive Wannier functions in (9) is $\pi$ the superfluid state was termed antiferromagnetic, while a zero phase difference characterizes ferromagnetic superfluid states. In Ref. [12], it was also predicted that ballistic expansion of an antiferromagnetic or ferromagnetic superfluid state would render dissimilar time-of-flight measurements. Although, in principle, measuring the atomic population inversion will determine the character of the superfluid states, in this section we analyze the corresponding timeof-flight scenario in our model. In other words, we investigate if the different phase modulation of the superfluid states is sufficient for separating between the two possible states.

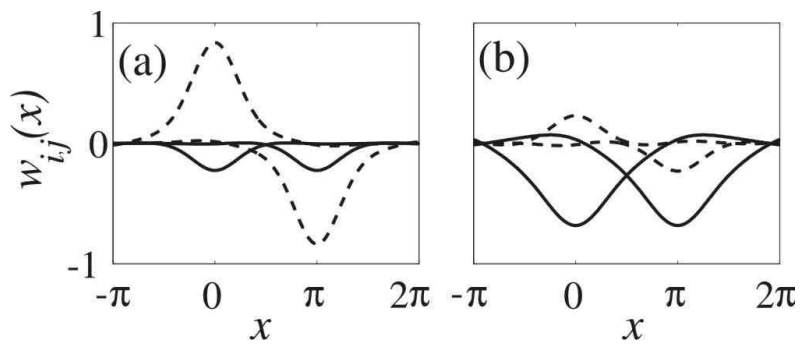

FIG. 5: Neighboring constituent Wannier functions $w_{j, 1}(x)$ (dotted) and $w_{j, 2}(x)$ (solid). In (a) $J_{1} \approx 0.1$ corresponding to $U=1, U_{1}=0.5, \Delta=-11.5$, while $J_{1} \approx-0.1$ in (b) obtained by choosing $U=1, U_{1}=0.5, \Delta=11.5$. Note that the dashed but not the solid line flip phase between neighboring sites. All parameters are dimensionless.

\section{A. Effect of positive and negative hopping coefficients}

In the internal $\{|1\rangle,|2\rangle\}$ basis, the spinor Wannier function at site $i$ is decomposed as

$$
\mathbf{w}_{j}(x)=\left[\begin{array}{l}
w_{j, 1}(x) \\
w_{j, 2}(x)
\end{array}\right] .
$$

Fig. 5 visualizes two examples of the neighboring Wannier functions. In (a), the nearest neighbor tunneling coefficient is positive, $J_{1} \approx 0.1$, while in (b) it is negative but with the same amplitude, $J_{1} \approx-0.1$. The figure evidences the swapping of internal state populations between positive and negative hopping. Consequently, an internal state selective measurement distinguishes between antiferromagnetic or ferromagnetic states and between Mott "+" and Mott "-" states. Nonetheless, in the next subsection, we also show how a time-of-flight detection can tell apart the two different superfluid states.

\section{B. Time-of-flight detection}
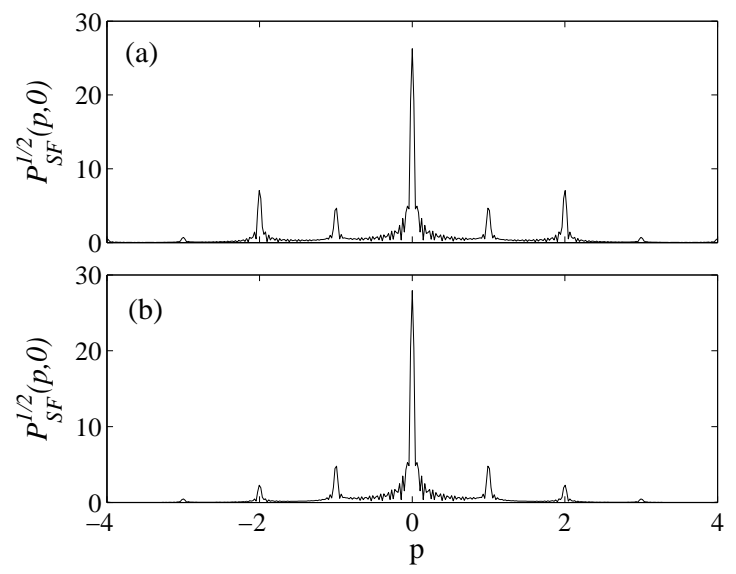

FIG. 6: The momentum distributions of the state (17) with $\Delta=-11.5$ (a) and $\Delta=11.5$ (b). The other dimensionless parameters are as in Fig. 5. The peaks at $p= \pm 2$ are more pronounced in (a) than in (b) giving different interference patterns of the freely expanding atomic condensates.

Our idea is to study the different interferences induced by having 0 or $\pi$ phase correlation between neighboring sites. Thus, we have a matter wave function as

$$
\Psi_{S F}(x, 0)=\sum_{j} e^{i \theta_{j}}\left[\begin{array}{l}
w_{j 1}(x) \\
w_{j 2}(x)
\end{array}\right]
$$

and study the impact of having

$$
\theta_{j}= \begin{cases}0, & J_{1}>0 \\ j \pi, & J_{1}<0\end{cases}
$$

when the wave function is freely evolving. The ballistically expanded wave function reads

$$
\Psi_{S F}\left(x, t_{t o f}\right)=e^{-i \hat{p}^{2} t_{t o f}} \Psi_{S F}(x),
$$

where $t_{\text {tof }}$ is the time-of-flight time between release of the superfluid state till measurement of it. The total probability distribution

$$
P_{t o t}\left(x, t_{t o f}\right)=\left|\Psi_{S F}\left(x, t_{t o f}\right)\right|^{2}
$$

or its constituent probability distributions $P_{i}\left(x, t_{t o f}\right)(i=$ $1,2)$ for the atomic internal states are assumed being detected after the ballistic expansion. For $t_{\text {tof }} \rightarrow \infty$, the momentum distribution $P_{S F}(p, t=0)$ corresponding to $\Psi_{S F}(x, 0)$ in Eq. (17), is encoded into the distribution $\Psi_{S F}\left(x, t_{t o f}=\infty\right)$. In Fig. 6, we display the square root 
of the momentum distributions of $\Psi_{S F}(x, 0)$. In (a) we use the parameters of Fig. 5] (a) (and thus a constant phase between the Wannier functions), while in (b) the parameters are as in Fig. 5] (b) ( $\pi$ phase modulation between the neighboring Wannier functions). For $J_{1}>0$, the even numbers of momenta are more strongly populated; the peaks around \pm 2 are more distinct in Fig. 6](a) than in (b). Note that for this example $J_{1}$ have the same strength in both cases, but the corresponding Wannier functions (see Fig. 5) are different. Thus, it is not only the phase $\theta_{j}$ which distinguishes the two cases.
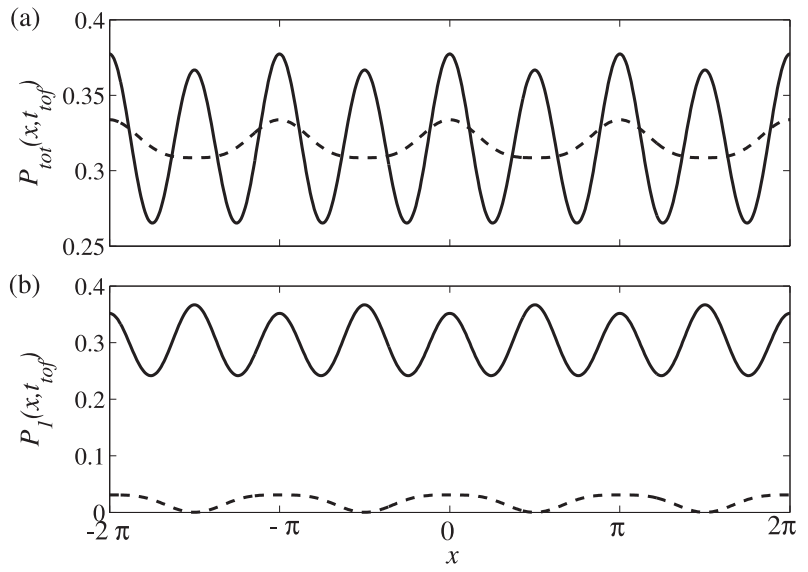

FIG. 7: Atomic distributions $P_{t o t}\left(x, t_{t o f}\right)\left(\right.$ a) and $P_{1}\left(x, t_{t o f}\right)$ (b) after a time-of-flight spreading $t_{\text {tof }}=4$. For solid lines; $\Delta=11.5$, while for dotted lines; $\Delta=-11.5$, and in both cases $U=1$ and $U_{1}=0.5$. This set of parameters give $J_{1}=0.1$ and $J_{1}=-0.1$ respectively. The difference between solid and dotted lines derives from the different Wannier functions of the two cases, but also from the phase factor $e^{i \theta_{j}}$ in the Wannier expansion (9).

The difference in momentum distributions will also manifest itself in the position distributions $P_{t o t}\left(x, t_{t o f}\right)$ and $P_{i}\left(x, t_{t o f}\right)$ for finite times $t_{t o f}$. The results for the total probability distribution (20) and the distribution for the internal state $1, P_{1}\left(x, t_{t o f}\right)$, are depicted in Fig. 7 (a) and (b) respectively. The time-of-flight $t_{t o f}=4$, guaranteeing that the interference has been well established. Noticeable from the figure is that for positive hopping the distribution shows a super-structure with two local maxima for each period, not seen for $J_{1}<0$. The great difference in probability amplitude between the internal atomic states in (b) derives from the fact that the two internal states are unequally populated due to the different detunings; $\Delta=11.5$ and $\Delta=-11.5$.

\section{CONCLUSIONS}

In this work we have presented an analysis of a gas of coupled two-level bosonic atoms in an one dimensional optical lattice. The spectrum of the single particle Hamiltonian was found to possess peculiar characteristics originating from the coupled dynamics. In an earlier work, we demonstrated that PTs can be obtained both for fermionic and bosonic atoms in the current model in the absence of atom-atom interaction [15]. Including scattering between the atoms, as in this paper, we identified the PT of Ref. [15] as a sign change in the site hopping parameter. The corresponding PT was shown to be between distinguishable Mott or superfluid states, characterized by an imbalance of the population of internal atomic states. Moreover, a thorough analysis about the effect of a positive or a negative nearest neighbor tunneling coefficient was given, focusing on time-of-flight detection of the condensate. The model can be generalized to two and three dimensions, and due to the anomalous dispersions found also in higher dimensions [15, 26], the corresponding phase diagrams is believed to show similar structures. However, in higher dimensions the strong coupling expansion method utilized in this work is supposed to give less reliable results, and other approaches would then be preferable.

The imposed approximations and their validity regimes were studied. The present paper restrict the analysis to regimes where these approximations are justified. However, it is expected that new phenomena will occur beyond such limitations, not encountered in the regular Bose-Hubbard Hamiltonian. For example, the nearest neighbor hopping may vanish, and consequently long range or semi-long range interaction might become important. We are currently investigating regimes outside single band and tight binding approximations by using different methods. These results are left for future publications. We are also studying the dynamics of the condensate as the system is driven through the critical point $J_{1}=0$ [26].

\section{Acknowledgments}

JL acknowledge support from the MEC program (FIS2005-04627).
[1] M. Lewenstein, A. Sanpera, V. Ahufinger, B. Damski, A. Sen, and U. Sen, Adv. Phys. 56, 243 (2007).

[2] M. Greiner, O. Mandel, T. Esslinger, T. W. Hänsch, and I. Bloch, Nature 415, 39 (2002).

[3] B. Juliette, V. Josse, Z. Zuo, A. Bernard, B. Hambrecht,
P. Lugan, D. Clément, L. Sanchez-Palencia, P. Bouyer, and A. Aspect, Nature 453, 891 (2008); G. Roati, C. D'Errico, L. Fallani, M. Fattori, C. Fort, M. Zaccanti, G. Modugno, and M. Inguscio, Nature 453, 895 (2008).

[4] B. Parades, A. Widera, V. Murg, O. Mandel, S. Fölling, 
I. Cirac, G. V. Schlyapnikov, T. W. Hänsch, and I. Bloch, Nature 429, 277 (2004).

[5] U. Schneider, L. Hackermüller, S. Will, I. Bloch, T. A. Costi, R. W. Helmes, D. Rasch, and A. Rosch, arXiv:0809.1464.

[6] E. Demler, and F. Zhou, Phys. Rev. Lett. 88, 163001 (2002); A. Imambekov, M. Lukin, and E. Demler, Phys. Rev. A 68, 063602 (2003); J. J. Garcia-Ripoll, M. A. Martin-Delgado, and J. I. Cirac, Phys. Rev. Lett. 93, 250405 (2005); T. Kimura, S. Tsuchiya, and S. Kurihara, Phys. Rev. Lett. 94, 110403 (2005); R. V. Pai, K. Sheshadri, and R. Pandit, Phys. Rev. B 77, 014503 (2008).

[7] O. Mandel, M. Greiner, A. Widera, T. Rom, T. W. Hänsch, and I. Bloch, Phys. Rev. Lett. 91, 010407 (2003).

[8] M. S. Chang, C. D. Hamley, M. D. Barrett, J. A. Sauer, K. M. Fortier, W. Zhang, L. You, and M. S. Chapman, Phys. Rev. Lett. 92, 140403 (2004).

[9] D. M. Stamper-Kurn, H. J. Miesner, A. P. Chikkatur, S. Inouye, J. Stenger, and W. Ketterle, Phys. Rev. Lett. 83, 661 (1999).

[10] L. E. Sadler, J. M. Higbie, S. R. Leslie, M. Vengalattore, and D. M. Stamper-Kurn DM, Nature 443, 312 (2006).

[11] A. Albus, A. Alluminati, and J. Eisert, Phys. Rev. A 68, 023606 (2003); H. P. Buchler, and G. Blatter, Phys. Rev. Lett. 91, 130404 (2003); M. Lewenstein, L. Santos, M. A. Baranov, and H. Fehrman, Phys. Rev. Lett. 92, 050401 (2004); K. Gunter, T. Stoferle, H. Moritz, M. Köhl, and T. Esslinger, Phys. Rev. Lett. 96, 180402 (2006).

[12] K. V. Kuritsky and R. Graham, Phys. Rev. Lett. 91, 240406 (2003).

[13] K. V. Kuritsky and R. Graham, Phys. Rev. A 70, 063610 (2004); K. V. Kuritsky, M. Timmer, and R. Graham,
Phys. Rev. A 71, 033623 (2005).

[14] J. J. Garcìa-Ripoll and J. K. Pachos, New J. Phys. 9, 139 (2007); M. Eckholt and J. J. Garcìa-Ripoll, Phys. Rev. A 77, 063603 (2008).

[15] J. Larson and J.-P. Martikainen, Phys. Rev. A 78, 063618 (2008).

[16] M. Alexanian and S. K. Bose, Phys. Rev. A 52, 2218 (1995).

[17] W. Ren and H. J. Carmichael, Phys. Rev. A 51, 752 (1995); J. Larson, J. Salo, and S. Stenholm, Phys. Rev. A 72, 013814 (2005).

[18] C. Wu and D. S. Sarma, Phys. Rev. B 77, 174509 (2008).

[19] A. S. Alexandrov and I. O. Thomas, J. Phys.: Condens. Matter 21, 245602 (2009); ibid, arXiv:0812.1977.

[20] M. P. A. Fisher, P. B. Weichman, G. Grinstein, and D. S. Fisher, Phys. rev. B 40, 546 (1989); S. Sachdev, Quantum Phase Transitions, (Cambridge University Press, 2006).

[21] J. Larson, S. Fernández-Vidal, G. Morigi, and M. Lewenstein, New J. Phys. 10, 045002 (2008).

[22] C. A. Maed, Rev. Mod. Phys. 64, 51 (1992); A. Bohm, A. Mustafazadeh, H. Kuizumi, Q. Niu, and J. W. Zwanziger, Geometrical Phase in Quantum Systems, (Springer, berlin, 2003).

[23] Defining a diabatic representation and diabatic limit in the present model is non-trivial.

[24] J. K. Freericks and H. Monien, Europhys. Lett. 26, 545 (1994).

[25] Discarding this term from the Hamiltonian gives a model in which the two atomic states are directly coupled by the optical lattice, see [15].

[26] A. Collin, J.-P. Martikainen, and J. Larson, in preparation. 